ISSN: $1130-3743$ - e-ISSN: 2386-5660

DOI: http://dx.doi.org/10.14201/teoredu2926186

\title{
CONSTRUCCIÓN DE CONOCIMIENTO COLABORATIVO MEDIADO TECNOLÓGICAMENTE: APORTACIONES TEÓRICAS DESDE EL ANÁLISIS DE PRÁCTICAS EDUCATIVAS
}

\section{Technology-mediated collaborative learning: theoretical contributions based on analysis of educational practice}

\section{Construction de la connaissance collaborative par le biais de la technologie: apports théoriques à partir de l'analyse des pratiques éducatives}

Sonia Casillas Martín, Marcos Cabezas GonzÁlez y Azucena Hernández Martín Universidad de Salamanca. Facultad de Educación. Departamento de Didáctica, Organización y Métodos de Investigación. C/ Paseo de Canalejas, 169.

37008Salamanca.scasillasma@usal.es; mcabezasgo@usal.es; azuher@usal.es

Fecha de recepción: enero de 2017

Fecha de aceptación: marzo de 2017

RESUMEN

El aprendizaje colaborativo ha sido objeto de un gran interés en el ámbito de la investigación educativa debido a las potencialidades del proceso de colaboración en el aprendizaje de los estudiantes, la construcción de sus conocimientos, el desarrollo de diversas capacidades y la mejora del rendimiento académico. A partir de una revisión conceptual y de la reflexión exhaustiva sobre este tema, nuestro trabajo presenta los resultados de un estudio de casos realizado en distintos centros educativos castellanoleoneses, para identificar pautas de actuación comunes, mediante la puesta en práctica de métodos de aprendizaje colaborativo mediados por el uso de las Tecnologías 
de la Información y la Comunicación (TIC). Entre los múltiples resultados ofrecidos, concluimos destacando la necesidad de planificar de forma muy cuidadosa el trabajo colaborativo aprovechando las posibilidades que ofrecen las TIC, como entornos comunicativos donde poder construir un conocimiento conjunto y compartido.

Palabras clave: aprendizaje colaborativo; Tecnologías de la Información y la Comunicación; tecnología educativa; innovación educativa; calidad de la educación.

\section{SUMMARY}

Collaborative learning has been a subject of great interest in the context of educational research, giving rise to many studies emphasizing the potential of the collaboration process in student learning, knowledge building, the development of diverse abilities and improved academic performance. Based on a conceptual review and thorough reflection on this topic, this article presents the results of a case study carried out in different schools in the Autonomous Community of Castile y Leon (Spain) in an attempt to identify patterns of common action through the implementation of collaborative learning methods mediated by information and communication technologies (ICT). Among the many outcomes of this study, we conclude by highlighting the need to plan collaborative work very carefully, taking advantage of the opportunities offered by ICT as communicative environments where it is possible to construct joint and shared learning.

Key words: collaborative learning; Information and Communication Technologies; educational technology; educational innovation; quality of education.

\section{SOMMAIRE}

L'apprentissage collaboratif a fait l'objet d'un grand intérêt dans le domaine de la recherche éducative, favorisant la mise en place de nombreuses études qui insistent sur le potentiel du processus de collaboration dans l'apprentissage des étudiants, l'acquisition de ses connaissances, le développement de certaines aptitudes et l'amélioration des résultats scolaires. Fondé sur une révision conceptuelle et une réflexion approfondie sur ce sujet, cet article présente les résultats d'une étude de cas réalisée dans différents centres éducatifs de la Communauté Autonome de Castille-et-León (Espagne) afin de cerner certaines normes de comportement commun générées par la mise en pratique des méthodes d'apprentissage collaboratif par le biais des technologies de l'information et de la communication (TIC). Parmi les multiples résultats obtenus, nous concluons en insistant sur la nécessité de planifier très soigneusement le travail collaboratif, en profitant des possibilités qu'offrent les TIC en tant qu'environnement de communication où peut se créer un apprentissage collégial et collectif.

Mots clés: apprentissage collaboratif; Technologies de l'Information et de la Communication; technologie éducative; innovation éducative; qualité de l'éducation. 
SONIA CASILLAS MARTÍN, MARCOS CABEZAS GONZÁLEZ Y AZUCENA HERNÁNDEZ MARTÍN

\section{INTRODUCCIÓN}

Nuestro trabajo da a conocer los resultados de una investigación que lleva por título «Aprendizaje colaborativo a través de las Tecnologías de la Información y Comunicación (TIC) en el contexto de la Escuela 2.0»', concluida en el año 2015.

A través de una metodología de estudio de casos, profundizamos en las características de los procesos que se generan en el aula con estas formas de acción pedagógicas en las que se enfatiza la colaboración entre iguales. Al mismo tiempo comprobamos si dichas acciones implican cambios en los resultados de aprendizaje, considerados en términos de competencias que obtienen los estudiantes.

Este artículo presenta, en primer lugar, los principales resultados del estudio de casos llevado a cabo en distintos centros educativos de la Comunidad de Castilla y León, con los objetivos principales de identificar algunas pautas comunes de actuación a partir del potencial educativo que posee la interacción colaborativa, y generar teoría desde el análisis de un conjunto de buenas prácticas educativas.

\section{ANTECEDENTES Y FUNDAMENTACIÓN TEÓRICA}

En los últimos años, el aprendizaje colaborativo ha sido objeto de un gran interés en el ámbito de la investigación educativa debido a las potencialidades del proceso de colaboración en el aprendizaje de los estudiantes, la construcción de sus conocimientos, el desarrollo de diversas capacidades y la mejora del rendimiento académico. Los resultados de todas estas investigaciones han propiciado paulatinamente la integración del aprendizaje colaborativo en el proceso de enseñanza como una estrategia muy adecuada para dar respuesta a las exigencias de la sociedad actual y, más concretamente, a las habilidades que deben poseer los estudiantes del siglo XxI.

El concepto de aprendizaje colaborativo responde a un enfoque constructivista y sociocultural, siguiendo las aportaciones teóricas de autores como Piaget y Vygotsky. Ambos sientan las bases de dicho aprendizaje al considerar, por una parte, que en el desarrollo del individuo no solo intervienen factores internos como la maduración y el desarrollo psicológico, sino también externos -el ambiente de aprendizaje, por ejemplo-. Y, por otra, al concebir el aprendizaje como un proceso social, resultado de la interacción entre las personas.

Johnson y Johnson (1987) y Johnson, Johnson y Smith (1998) nos ofrecen una caracterización ya clásica del aprendizaje colaborativo en la que se enfatiza la interdependencia que existe entre el esfuerzo y aprendizaje individual y el grupal, ya que cada miembro del grupo es responsable tanto de su aprendizaje, como del de los restantes miembros. Y en la motivación para ayudarse mutuamente en aras de conseguir unos objetivos comunes. Por su parte, Lara (2001) define este

1. Investigación I+D financiada por el Ministerio de Ciencia e Innovación (EDU2011-28071). 
aprendizaje como una "filosofía» que implica y favorece el trabajo, la construcción, el aprendizaje, el cambio y la mejora de forma conjunta.

En la actualidad la puesta en práctica de formas de aprendizaje colaborativo en el aula y la adquisición de toda una serie de competencias conceptuales, procedimentales y actitudinales en los estudiantes se ven impulsadas por el empleo de las herramientas tecnológicas. La Web 2.0, por ejemplo, constituye un buen aliado para construir conocimientos de forma colaborativa (De la Torre, 2006; Cebrián, 2008; Cabezas y Casillas, 2009; Zabala y Arnau, 2009), al enfatizar un nuevo modelo comunicativo en el que priman los procesos de interrelación entre personas o grupos, mediante la utilización de una gran cantidad y variedad de herramientas con las que gestionar contenidos e información.

La metodología de aprendizaje colaborativo apoyado en el uso de las tecnologías ha tenido un fuerte desarrollo en el ámbito educativo. Toda la investigación generada en los últimos años sobre el CsCL (Computer Suported Collaborative Learning) ha permitido el desarrollo de un nuevo paradigma que ha logrado relacionar las principales teorías del aprendizaje con los recursos tecnológicos, desde una perspectiva sociocultural de la cognición. E incide en la idea de que los procesos de aprendizaje poseen un carácter eminentemente social, por lo que se considera que las Tecnologías de la Información y la Comunicación (TIC) son herramientas enriquecedoras para crear contextos interpersonales de aprendizaje (Col, Maurí y Onrubia, 2008; Salmerón, Rodríguez y Gutiérrez, 2010).

La investigación sobre el aprendizaje colaborativo mediado tecnológicamente destaca sus efectos positivos en los contextos de enseñanza y aprendizaje. Los estudios más clásicos realizados al respecto por diversos autores (Maini y Comoglio, 1995; Lou, Abrami, Spence, Poulsen, Chambers y D'Apollonia, 1996; Springer, Stanne y Donovan, 1999) con estudiantes de distintos niveles educativos evidencian que trabajar colaborativamente favorece el desarrollo de competencias para la autonomía personal, activa habilidades sociales concretas y mejora el rendimiento académico del grupo. Considerando este último aspecto, dichos estudios ponen de manifiesto además que el rendimiento académico de los estudiantes que trabajan en grupo supera al de los que realizan el trabajo de forma individual. Por otra parte, también se destaca que en contextos de aprendizaje colaborativo los estudiantes generan actitudes positivas hacia los demás y un mejor autoconcepto de sí mismos.

Los resultados de otras investigaciones más cercanas en el tiempo, como la que nos plantean Arancibia, Carcamo, Contreras, Scheihing y Troncoso (2014), revelan que las experiencias colaborativas son objeto de una valoración alta. Concretamente, este estudio destaca los efectos motivadores que el empleo de herramientas tecnológicas genera en los estudiantes, el interés por colaborar con alumnos de otros centros educativos en experiencias concretas, así como la variedad de interacciones que se generan entre ellos. Por tanto, el conocimiento que se genera en un contexto colaborativo se ve enriquecido por la mediación tecnológica. En este sentido, Zañartu-Correa (2013) señala que las experiencias de aprendizaje colaborativo mediadas por la tecnología permiten que dos o más 
personas construyan conocimiento, a través de la discusión, la reflexión y la toma de decisiones en un contexto en el que los distintos recursos informáticos actúan como mediadores. «Este proceso social trae como resultado la generación de conocimiento compartido, que representa el entendimiento común de un grupo con respecto al contenido de un dominio específico» (p. 9).

En nuestro país contamos también con investigaciones recientes y muy interesantes sobre este tema. Domingo, Sánchez y Sancho (2014) realizaron un estudio con alumnado del último curso de Educación Secundaria Obligatoria, con el objetivo de explorar cómo y con qué se comunican, expresan y aprenden los adolescentes dentro y fuera de las aulas. Entre las múltiples dimensiones consideradas se debatió sobre el aprendizaje colaborativo y las potencialidades de los recursos digitales, constatándose que trabajar colaborativamente empleando dichos recursos favorece la construcción de conocimientos y aprendizajes no incluidos en el currículo, a la vez que genera experiencias muy ricas para conectar y dotar de sentido a la información. Buscar información, analizarla, compartirla, editarla; $y$, muy especialmente, aprender a gestionar los propios procesos de aprendizaje no aisladamente, sino en interacción con los demás, permite compartir objetivos, distribuir responsabilidades, solucionar conjuntamente problemas, potenciando el rol que pueden desempeñar las herramientas tecnológicas en todo el proceso.

Los estudios efectuados con docentes de centros de Educación Primaria y Secundaria ponen de relieve también que el profesorado identifica más ventajas que inconvenientes en el aprendizaje colaborativo mediado por el empleo de la Tecnología (García-Valcárcel y Tejedor, 2010; Suárez y Gros, 2013; García-Valcárcel, Basilotta y López, 2014). Las principales aportaciones se relacionan con el desarrollo de competencias transversales, la interacción entre alumnos, la motivación, la asunción de responsabilidades, la capacidad para reflexionar y tomar iniciativa en distintas situaciones y la mejora del aprendizaje de alumnos con necesidades educativas especiales.

Aunque las ventajas de trabajar colaborativamente son manifestadas por el profesorado, el hecho de que posteriormente su puesta en práctica sea todavía puntual y circunscrita a situaciones muy concretas se debe en gran medida a diversas limitaciones implícitas en la puesta en práctica de estas formas de trabajo, utilizando recursos tecnológicos. Entre ellas se apunta, por ejemplo, la mayor complejidad que supone para los docentes repensar formas de trabajo colaborativo por medio de la utilización de dichas herramientas, al ser necesarios el diseño y la organización cuidadosos de las actividades, así como la previsión de problemas de comunicación de diverso tipo que pueden generarse entre los estudiantes durante su desarrollo. Otras dificultades a las que se refieren distintos estudios se relacionan con la necesidad de un tiempo adicional del que en ocasiones no dispone el profesorado para gestionar la comunicación en Internet (Ferro, Martínez y Otero, 2009; Monereo y Badía, 2012; Suárez y Gros, 2013). En definitiva, y como ponen de relieve otros autores, más allá de estas limitaciones, las situaciones de aprendizaje planteadas como una interacción de carácter 
colaborativo implican un cambio en «la forma de representar y estructurar la interacción entre alumnos» (Suárez, 2008, 113).

\section{DisEÑO Y METODOLOGÍA}

Este artículo ha centrado su atención en presentar en profundidad los resultados de los diversos proyectos sobre trabajo colaborativo mediados por el empleo de las TIC, llevados a cabo en centros con altas prestaciones tecnológicas en la Comunidad de Castilla y León; a través de un seguimiento de las actividades realizadas, los procesos de aprendizaje que se estimulan y la adquisición de competencias por parte de los alumnos. Para ello, se han tenido en consideración las reflexiones de los profesores a lo largo del desarrollo del proyecto, las observaciones de los investigadores, la opinión de los estudiantes sobre la acción pedagógica seguida y los resultados obtenidos. Todo ello por medio de una metodología de investigación de estudios de casos múltiples, fijando en ocho los casos sobre los que se realizó el seguimiento. Planteamos dicho estudio de casos múltiples o colectivo porque nuestra pretensión era comparar, con un protocolo común, distintos centros educativos en los que se realizan experiencias de aprendizaje colaborativo utilizando distintas herramientas tecnológicas; todo ello con objeto de llegar a un cierto grado de generalización naturalista, basado en la determinación de las pautas comunes que surgen en los casos analizados y en el reconocimiento de las similitudes y diferencias de las situaciones descritas. Aunque, como bien se sabe, la metodología de estudio de casos no pretende llegar a generalizaciones, al no tratarse de una investigación de muestras (Stake, 2005), consideramos que sí es posible generar teoría desde la propia práctica mediante la adopción de una perspectiva reflexiva sobre lo acontecido en situaciones concretas.

Se ha definido el caso como un proyecto educativo en el que se trabaja de forma colaborativa y se utilizan herramientas tecnológicas para llevarlo a cabo.

En el estudio de casos, la formulación de las preguntas de investigación es una tarea fundamental. En este trabajo planteamos unas cuestiones que dirigieron nuestra atención y reflexión posterior. Las preguntas temáticas que nos planteamos están relacionadas con los siguientes aspectos:

- El grado de adecuación en los centros educativos respecto a las infraestructuras, el profesorado y la organización escolar para desarrollar proyectos de trabajo colaborativo utilizando TIC.

- La adquisición por parte de los estudiantes de las competencias deseadas, tanto curriculares como personales e interpersonales.

- La potencialidad de las estrategias de aprendizaje colaborativo para favorecer a todos los estudiantes.

- Las posibles adaptaciones de la evaluación del trabajo colaborativo para valorar el nivel de aprendizaje de cada estudiante. 
Es importante no confundir estos temas con las preguntas informativas, las cuales buscan información para la descripción del caso, no tienen que estar vinculadas a organizadores conceptuales y se pueden utilizar como estructura conceptual para la presentación del mismo. Si bien se podrían subordinar a la estructura temática. Así, nos planteamos dichas preguntas informativas tomando como criterio tres grandes ejes: el contexto, la acción pedagógica desarrollada y la evaluación.

Respecto al primero de ellos, el contexto, se pretendió indagar cómo afectaba el contexto social al desarrollo de propuestas de trabajo colaborativo; qué características tenían el centro y el aula o aulas donde se desarrollaba el proyecto; de qué recursos disponían en el centro, en el aula ordinaria y en los hogares; cuántos profesores y alumnos estaban implicados en el proyecto y qué les definía. Y, por último, qué apoyo tuvo este profesorado del equipo directivo y de otros compañeros.

En lo que concierne a la acción pedagógica desarrollada, nos resultó interesante investigar en qué nivel/es educativo/s se desarrolló el proyecto; qué finalidades se planteaban; áreas curriculares y contenidos concretos trabajados; organización de los tiempos y espacios; planificación de las sesiones, actividades y recursos empleados; roles desempeñados por los alumnos y el profesor; y grado de apoyo de las familias.

Por último, en cuanto a la evaluación, se pretendió recoger información acerca de qué tipo de valoración se llevaba a cabo sobre el aprendizaje de los alumnos, la calidad de las tareas realizadas, la utilidad de los recursos digitales, la valoración del aprendizaje por parte de los alumnos, las competencias de aprendizaje que se vieron reforzadas y a qué estudiantes les favoreció esta forma de trabajo. En el siguiente gráfico resumimos la secuencia considerada en los estudios de casos: 


\section{GRÁFICO 1}

SECUENCIA CONSIDERADA EN LOS ESTUDIOS DE CASOS

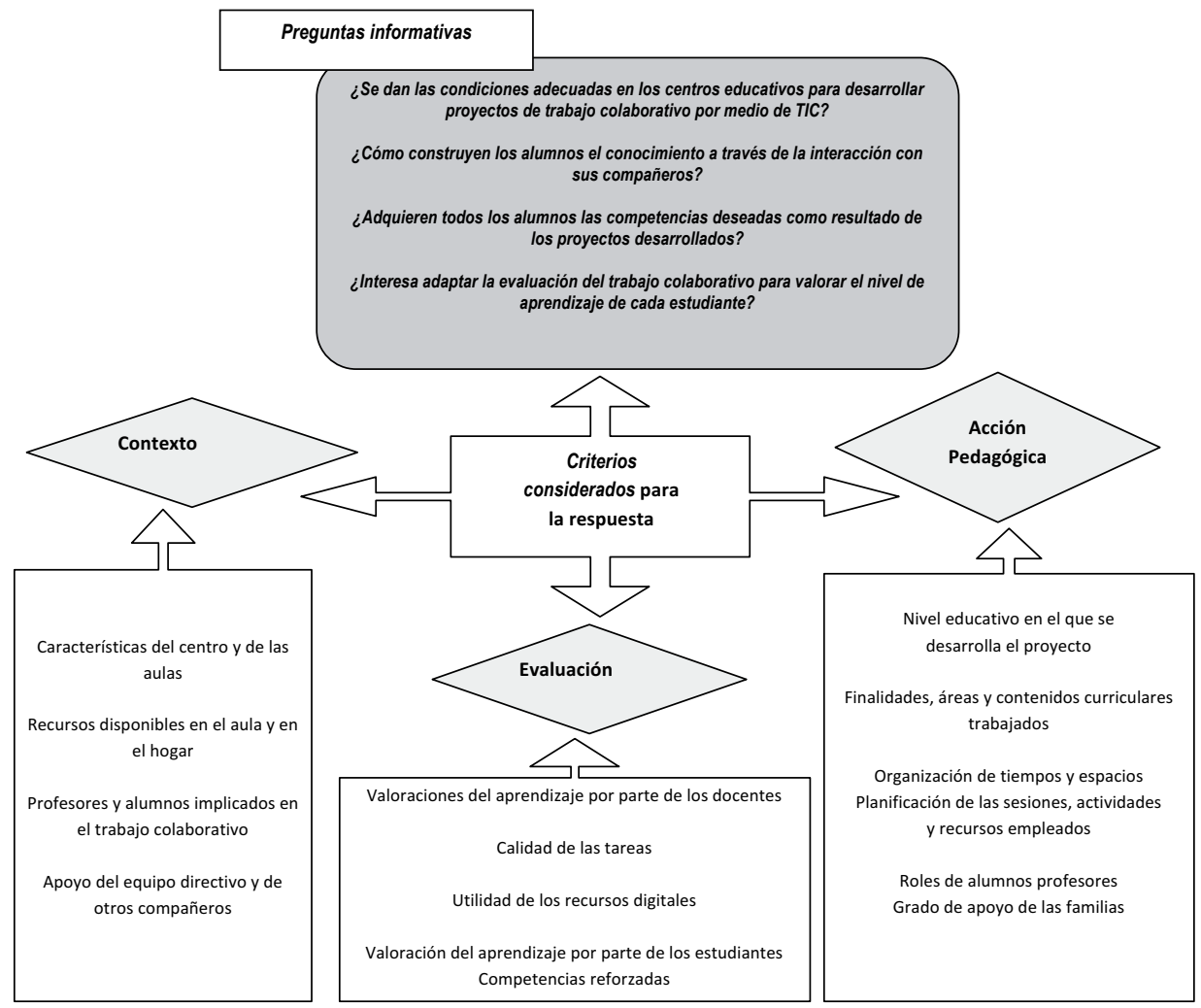

\subsection{Muestra. Selección de los casos de estudio: proyectos de aprendizaje colaborativo con TIC}

Contamos con un contexto de estudio diverso atendiendo a la disponibilidad de los centros educativos para participar en el proceso de investigación. Dispusimos de un universo amplio, y en cierto modo heterogéneo, que nos permitió ver distintas realidades respecto al lugar en el que se encuentran, la titularidad de los centros y la etapa o etapas educativas en las que se imparte la docencia.

En total se seleccionaron ocho proyectos de diferentes centros educativos, constituyendo cada uno de ellos un caso. Los criterios para la selección de los ocho casos, siguiendo a Stake (2005), fueron:

1. La rentabilidad de aquello que aprendemos (oportunidades de aprendizaje). 
SONIA CASILLAS MARTÍN, MARCOS CABEZAS GONZÁLEZ Y AZUCENA HERNÁNDEZ MARTÍN

2. La facilidad de abordar (nuestras indagaciones son bien acogidas, los actores o personas estudiadas están dispuestas a colaborar, dar su opinión y dedicar el tiempo preciso).

3. El equilibrio y la variedad (estudio de casos en diversas provincias, niveles educativos y ámbitos rural/urbano).

A excepción de un colegio situado en Madrid, todos los demás fueron centros rurales ubicados en distintas zonas de Castilla y León (Ávila, Salamanca, Zamora, Segovia, Palencia, Valladolid y León). La titularidad de los mismos fue fundamentalmente pública, salvo en uno de ellos, con carácter privado-concertado, y las etapas educativas eran diversas, aunque predominaba la de Educación Infantil y la de Educación Primaria. En cuanto a los tipos de centros, seis eran de Infantil y Primaria (CEIP), uno de Educación Obligatoria (CEO), un Centro Rural Agrupado (CRA), un Centro de Formación Profesional (FP) y un Instituto de Educación Secundaria (IES). Los profesores mostraron en todo momento su máxima colaboración con la investigación y una buena disposición para llevar a cabo las actividades propuestas de recogida y análisis de la información.

Señalar que tres de los ocho casos contaban con la certificación TIC de nivel cinco para centros educativos en aplicación de Tecnologías de la Información y la Comunicación, que es otorgada por la Consejería de Educación de Castilla y León. Ello indica que disponían de equipamiento tecnológico e infraestructuras adecuadas, proyectos de innovación activos relacionados con la implantación de las TIC y una formación adicional en herramientas tecnológicas que se ofrecía al profesorado.

En todo momento, los procedimientos utilizados en los muestreos y controles fueron realizados tras la obtención de un consentimiento informado.

La muestra que participó en la investigación estuvo compuesta por un total de 19 docentes y 253 estudiantes.

Si analizamos en conjunto el contexto en el que se desarrolló nuestra investigación, a partir del estudio de cada uno de casos, podemos señalar que se trataba de centros con infraestructura y recursos diversos, de acuerdo al tamaño, las etapas en las que se imparte docencia, su trayectoria con el empleo de las Tic y la participación a lo largo de los diferentes cursos en proyectos colaborativos. De modo prácticamente generalizado, había un ordenador y un proyector en el aula ordinaria y en alguna de ellas también una pizarra digital. En casi todas se disponía de conexión a Internet mediante wifi, aunque en otras no se contaba con materiales digitales, por lo que se empleaba el aula de informática, a disposición de todo el colegio. Fue significativa la utilización de equipos para gestionar informáticamente los préstamos en las bibliotecas de algunos de los colegios. En aquellos centros en donde venía siendo más habitual la implementación de metodologías de aprendizaje colaborativo mediado por TIC, había una mayor disposición de recursos para llevar a cabo la integración de estas herramientas, independientemente del nivel o la especialidad. Por tanto, se observa que, con relación a las características de los centros y de las aulas, existe un contexto más o menos propicio para el desempeño 
de trabajos que generen un conocimiento colaborativo mediado tecnológicamente. Al tratarse, en algunos casos, de centros con una especial sensibilidad hacia el trabajo colaborativo y el empleo de las herramientas tecnológicas en su desarrollo, el apoyo por parte del equipo directivo a aquellos profesores que realizaban estas experiencias fue importante. Por el contrario, en prácticamente todos los casos, a excepción de dos, evidenciamos que el apoyo de las familias en el desarrollo de las actividades planteadas con una metodología de aprendizaje colaborativo fue mínimo, bien porque las competencias de ciertos padres son limitadas o porque los progenitores consideraban que este tipo de actividades podía ir en detrimento del avance en los contenidos que se encuentran en los libros de texto.

\subsection{Instrumentos y técnicas para la recogida de información}

Los instrumentos empleados para la recogida de la información fueron la entrevista, la observación, el análisis de documentos, una guía de seguimiento para el profesor y un cuestionario de evaluación para los alumnos. Se combinaron distintas técnicas de naturaleza tanto cualitativa como cuantitativa.

La entrevista con el docente se realizó al inicio y al final del desarrollo del proyecto; en la inicial se recogió información sobre los objetivos del proyecto, las acciones pedagógicas desarrolladas, las expectativas del profesor, la planificación del proceso de aprendizaje y se explicó el procedimiento para realizar el seguimiento. En la entrevista final se efectuó una valoración global, recopilando datos sobre el contexto, las competencias profesionales desarrolladas y la evaluación de los resultados. Y se sometieron a consideración del docente las principales interpretaciones realizadas por el investigador.

La guía de seguimiento fue diseñada para que el profesor describiera y evaluase el proyecto, pudiéndose utilizar con un carácter semanal, quincenal o mensual, en función del tiempo de desarrollo del mismo. Los criterios considerados en dicha guía se referían a los objetivos y contenidos propuestos, las actividades realizadas, los materiales utilizados, los espacios, el agrupamiento de alumnos y el sistema y criterios de evaluación del aprendizaje. Finalmente se utilizó la rúbrica de Medina y Robles (2012) para evaluar el trabajo en grupos.

El registro de observación se elaboró con objeto de pautar las observaciones realizadas en los centros por los investigadores, y permitió recoger información descriptiva de forma abierta, además de valoraciones cuantitativas sobre algunos indicadores. Este registro facilitó, por ejemplo, la recogida de información sobre:

- Las características del centro y del aula donde se desarrolló la actividad académica.

- La contextualización y planificación de la sesión.

- La calidad de las tareas llevadas a cabo.

- Los roles de los alumnos y del profesor.

- El seguimiento y evaluación de las actividades realizadas. 
- La calidad de los logros académicos.

- La motivación y participación de los estudiantes.

- El clima de clase.

- Las competencias adquiridas.

Se elaboró un cuestionario dirigido a los alumnos de 10 a 14 años que participaron en los distintos proyectos, con la finalidad de recoger información sobre las materias trabajadas, los métodos y recursos tecnológicos empleados, la participación de la familia para ayudar en las tareas planteadas y la valoración del proceso y resultados de aprendizaje. Se empleó el formato de diferencial semántico, por resultar más sencillo y apropiado para la edad de los estudiantes. Dicha escala fue aplicada previamente a un grupo piloto de 140 alumnos de Primaria para su estudio psicométrico, depurando una escala inicial de 31 ítems. La escala final resultante de 20 ítems, con una graduación de 1 a 7, presentó una fiabilidad de 0,908. El análisis factorial realizado puso de manifiesto la presencia de cuatro factores: satisfacción con el aprendizaje, organización del trabajo, creación y colaboración, y comprensión de la actividad (García-Valcárcel y Basilotta, 2013).

\subsection{Procedimiento metodológico para realizar el trabajo de campo}

En relación a la recogida de información, los investigadores pusimos especial cuidado en no obstaculizar la actividad cotidiana y no examinar, respetando los principios de empatía y no intervencionismo. Empleamos la observación directa y la revisión de los datos y materiales recogidos, y se involucró a los actores (profesores y alumnos) en la recogida de información y la reflexión sobre la práctica. Para ello, se distribuyeron los ocho casos seleccionados entre los distintos miembros del equipo investigador.

Elaboramos un protocolo de actuación en el cual se especificó con detalle el procedimiento a seguir en el trabajo de campo, con el fin de mantener los mismos criterios en el contacto y acceso a los centros por parte de todos los investigadores. Establecimos un primer encuentro con el equipo directivo y con los docentes para explicarles los objetivos de la investigación, la planificación de las distintas sesiones de observación y la realización de entrevistas. Y, posteriormente, se concretó un procedimiento consensuado sobre la forma de proceder y hacer partícipe al profesorado del proceso de seguimiento y evaluación de su práctica profesional. De este modo, los profesores colaboradores tuvieron un papel muy activo, y apostaron por una metodología dialógica que implicaba la comunicación igualitaria entre investigadores e investigados.

Como estrategia para la validación de los resultados se empleó la triangulación, de modo que se redujeran al mínimo falsas representaciones e interpretaciones. Hemos de considerar que se puede emplear la triangulación de las fuentes de datos para comprobar si se mantiene el significado de lo que observamos cuando lo encontramos en otras circunstancias (otros momentos, espacios, cuando las 
personas interactúan de forma diferente...). Por otra parte, está la triangulación del investigador que supone ver si las observaciones de distintos investigadores sobre la misma escena o fenómeno llevan a la misma interpretación. Y, en tercer lugar, contamos con la triangulación metodológica que se basa en el uso de métodos múltiples como la observación, la entrevista, la revisión de documentos, etc., posibilitándonos puntos de vista de observación independientes. En nuestro estudio tomamos en consideración estas tres perspectivas para la validación de los datos e interpretaciones realizadas.

Efectuamos un «Cross-Case Análisis» o análisis de cruce de datos, método cualitativo utilizado cuando la unidad de análisis es el caso, y que permite examinar temas, similitudes y diferencias.

\section{PRESENTACiÓN Y Discusión De RESUlTados}

Debido a la gran cantidad de información recogida en los estudios de los ocho casos analizados, decidimos organizar la misma tomando como criterio dos de los aspectos considerados durante el análisis de cada uno de los casos: la acción pedagógica desarrollada y la evaluación.

Como ya hemos señalado, somos conscientes de que, al realizar este análisis en centros con características y matices diversos, no es posible generalizar, y menos cuando la metodología de investigación utilizada ha sido el estudio de casos. Sin embargo, la revisión exhaustiva de cada uno de los proyectos estudiados sí nos permite plantear algunos criterios comunes de actuación sobre el tema objeto de estudio, la construcción de conocimiento colaborativo mediada tecnológicamente. Nos interesa, en definitiva, proporcionar lo que algunos autores denominan generalizaciones naturalistas (Stake, 2005), aludiendo a que las personas pueden aprender muchas cosas generales de los casos particulares; y tratando de desarrollar, como ya señalamos, bases teóricas sobre el tema a partir del análisis de las situaciones prácticas.

Los resultados que presentamos se estructuran teniendo en cuenta los once tópicos considerados para elaborar los informes específicos de cada uno de los casos (Tabla 1). 
SONIA CASILLAS MARTÍN, MARCOS CABEZAS GONZÁLEZ Y AZUCENA HERNÁNDEZ MARTÍN

TABLA 1

TÓPICOS CONSIDERADOS EN LA ELABORACIÓN DE LOS INFORMES DE CADA CASO

\begin{tabular}{|c|c|}
\hline \multicolumn{2}{|c|}{ TópICOS } \\
\hline ACCIONES PEDAGÓGICAS DESARROLLADAS & EVALUACIÓN \\
\hline Las finalidades de los proyectos estudiados & El sistema de evaluación del aprendizaje \\
\hline La organización de espacios y tiempos & $\begin{array}{c}\text { La calidad de las tareas e interés } \\
\text { de los recursos digitales }\end{array}$ \\
\hline $\begin{array}{c}\text { Las tareas realizadas y los } \\
\text { recursos utilizados }\end{array}$ & $\begin{array}{c}\text { La valoración del proyecto por } \\
\text { parte de los alumnos }\end{array}$ \\
\hline $\begin{array}{c}\text { Los roles adoptados por los } \\
\text { profesores y los alumnos }\end{array}$ & $\begin{array}{c}\text { La valoración del proyecto por } \\
\text { parte de los profesores }\end{array}$ \\
\hline $\begin{array}{c}\text { El grado de apoyo de las familias en el } \\
\text { desarrollo de los proyectos colaborativos }\end{array}$ & $\begin{array}{c}\text { Las competencias adquiridas } \\
\text { por los estudiantes }\end{array}$ \\
\hline & Las competencias profesionales \\
de los docentes
\end{tabular}

\subsection{Las finalidades de los proyectos colaborativos}

Estas finalidades son muy diversas en función de las características de los proyectos trabajados en cada uno de los ocho casos, aunque en todos ellos encontramos tres que consideramos fundamentales:

- Introducir una filosofía de aprendizaje que, apoyada en la interacción colaborativa, permita el reparto de tareas y compartir información, priorizando un aprendizaje por descubrimiento y más experiencial.

- Desarrollar la competencia digital mediante el empleo de diversos recursos informáticos.

- Identificar las ventajas e inconvenientes del método de trabajo colaborativo en el aprendizaje de los alumnos.

Considerando la posibilidad que nos brinda este trabajo para extraer aportaciones teóricas desde los ámbitos prácticos en los que nos situamos, podemos señalar que los proyectos colaborativos tienen como denominador común el explorar las posibilidades del aprendizaje colaborativo mediado por el empleo de las Tic en el contexto educativo. Además, evidenciamos que esta forma de trabajar supone una nueva manera de entender el proceso de enseñanza que supera, o por lo menos diversifica, el uso del libro de texto; y, al mismo tiempo, se apuesta por una reorientación del proceso de aprendizaje, que contribuye a mejorarlo. En este sentido se pronuncia Suárez $(2008,376)$, cuando señala que "La presencia del "otro" no significa un impedimento para el aprendizaje, sino todo lo contrario (una oportunidad para su progreso)». El hecho de que sean ya bastantes los profesores que se plantean como objetivo el poner en práctica en sus aulas esta forma de trabajo se 
encuentra refrendado por numerosos estudios, en los que se evidencia la relación existente entre los métodos colaborativos y la mejora del proceso de aprendizaje (Doppenberg, Bakxy y Brok, 2012; García-Valcárcel, Hernández y Recamán, 2012; García-Valcárcel, Basilotta y López, 2014).

\subsection{La organización de espacios y tiempos}

Los espacios y los tiempos se organizan siguiendo los criterios del número y características de los alumnos, los recursos disponibles y la propia naturaleza de las actividades a realizar. En cuanto a la organización del espacio, se utiliza generalmente el aula ordinaria para explicar el trabajo a los estudiantes y la sala de informática para desarrollar las tareas utilizando ordenadores, tabletas y la pizarra digital interactiva (siempre y cuando en el aula ordinaria no se disponga de ellos). La organización del tiempo es variable en función de los objetivos perseguidos en cada uno de los casos.

El aprendizaje colaborativo exige, en definitiva, un ritmo de trabajo más dilatado en el tiempo; de modo que el alumnado pueda reflexionar, establecer consensos y llegar a la consecución de productos conjuntos, fruto del compromiso y la responsabilidad de cada uno de los miembros del grupo.

\subsection{Tareas realizadas y recursos empleados}

Mayoritariamente se combinan las exposiciones del profesor al gran grupo (generalmente, en las primeras fases de desarrollo del proyecto), en las que se explican la teoría, las instrucciones y la dinámica de trabajo, con el desarrollo de tareas en pequeños grupos para realizar las actividades concretas que supone cada proyecto.

Un hecho común que caracteriza todos los casos es la participación de los estudiantes en el desarrollo de actividades que exigen competencias informacionales y digitales (investigación, selección de información y empleo de recursos tecnológicos diversos). Destacar como actividad realizada en muchos de los casos en los que participaban varios centros la elaboración de un blog colaborativo con el objetivo de compartir los trabajos realizados por los alumnos.

Los recursos empleados son distintos, dependiendo del proyecto en cuestión y de la dotación de los diferentes centros. Aunque algunos son comunes en todos ellos: los libros de texto y otras lecturas, vídeos, Internet, ordenadores de sobremesa y miniportátiles, tabletas, pizarras digitales interactivas, cámaras de fotos digitales, webcam, pendrive, software variado (procesadores de texto, programas de presentación, navegadores, etc.) y almacenamiento en la nube (Dropbox, OneDrive, google drive, box, etc.).

La tecnología es, por tanto, una constante en los procesos de aprendizaje colaborativo puestos en marcha en todos los casos, tratando de desarrollar procesos de 
innovación educativa. Debemos recordar que la colaboración mediada por los distintos recursos tecnológicos enfatiza el rol que estos pueden ejercer como elementos mediadores en el proceso de aprendizaje. Como ya se ha manifestado en otro momento, actualmente contamos con numerosas herramientas que se incluyen, en su mayoría, dentro de la llamada Web 2.0, y cuyas virtualidades para diseñar o implementar métodos de aprendizaje colaborativo en otros contextos distintos a los presenciales son indudables (Hernández y Quintero, 2009).

\subsection{Roles adoptados por profesores y alumnos}

En todos los casos se evidencia un cambio de roles tanto en los docentes como en los estudiantes.

Es constante la alusión de los profesores a su papel de motivadores, guías y orientadores en el desarrollo de las actividades, con el apoyo y la resolución de dudas. Este aspecto lo evidencian también otros estudios más clásicos, como el realizado por Moreno y Martínez (2007), quienes señalan las preocupaciones de los docentes por posicionarse como facilitadores del aprendizaje, dejando una mayor autonomía a los alumnos para que realicen las tareas de clase y cooperen con los compañeros. Como consecuencia de todo ello, existe una mayor comunicación entre los alumnos y entre estos y el docente. Además, se presta una atención más individualizada a los estudiantes que tienen mayores necesidades.

Respecto a los alumnos, se muestran más participativos y, en palabras de sus profesores, asumen una gran responsabilidad porque se convierten en los verdaderos protagonistas de las tareas. Al mismo tiempo son conscientes de que la calidad del producto final dependerá del proceso de trabajo realizado en el grupo.

Este cambio de roles es un proceso más o menos lento valorado de forma muy positiva por los docentes, aspecto que se recoge también en diferentes investigaciones, en las que se exponen las potencialidades de la metodología de aprendizaje colaborativo para favorecer la asunción de roles que mejoren los procesos de enseñanza y aprendizaje (Javornik y Grmek, 2007; Shahzad, Valcke y Bahoo, 2012; Vázquez-Bernal, Wamba, Jiménez-Pérez y Lorca, 2010).

\subsection{Apoyo de las familias en el desarrollo de los proyectos colaborativos}

El rol que muchas familias siguen otorgando a la escuela es fundamentalmente el de transmisora de información, con el monopolio de un saber de corte academicista. Este rol atribuido tradicionalmente a los centros educativos entra en confrontación, en ocasiones, con otras formas de trabajo que, desde perspectivas más innovadoras, impliquen activamente la colaboración de los padres. Y, en consecuencia, sean percibidas por estos como una distorsión pedagógica que no redunda en el aprendizaje de sus hijos. A día de hoy sigue observándose una disociación importante entre el papel educativo que ejerce la familia y el que se 
considera que han de desempeñar las instituciones educativas formales. Además, hemos de tener en cuenta que, debido a cambios en la estructura y funcionamiento del núcleo familiar, se exige socialmente que los roles que tradicionalmente desempeñaban los progenitores y otros miembros del entorno familiar sean asumidos por los docentes. Todos estos factores pueden explicar el predominio de una escasa colaboración de los padres en el desarrollo de los proyectos colaborativos, tal como manifiestan los propios docentes en el estudio realizado.

\subsection{Sistema de evaluación del aprendizaje}

La evaluación del aprendizaje, tal como se desprende del análisis efectuado, es una tarea del profesor, si bien, en algunos casos también participan los alumnos por medio de la autoevaluación y, en menor medida, la coevaluación; ambas llevadas a cabo por medio de rúbricas diseñadas por los docentes. Estas diversas formas de evaluar permiten explorar nuevas maneras de ver y analizar las mismas acciones desde puntos de vista distintos.

Los principales criterios de evaluación que son tenidos en cuenta para valorar las tareas realizadas en cada uno de los proyectos se pueden resumir en las siguientes:

- Calidad del trabajo realizado.

- Grado de innovación y creatividad.

- Exhaustividad en la búsqueda de información.

- Motivación y esfuerzo.

- Grado de implicación en y del grupo.

- Corrección en la exposición escrita.

- Calidad de las técnicas de comunicación empleadas.

- Autonomía de los estudiantes para el manejo de dispositivos digitales (Kindle, IPad, portátiles, etc.).

Nos parece interesante destacar también que un gran número de profesores se decanta por una evaluación personalizada, adaptada a cada uno de los estudiantes, considerando sus características, necesidades e intereses; y, a la vez, por una evaluación continua, en la que se tengan en cuenta los avances individuales respecto a sus posibilidades, a sus conocimientos previos y a su competencia digital. Por otra parte, en la mayoría de los proyectos se realiza un seguimiento permanente de los trabajos efectuados, empleando la observación directa por parte del profesor para la recogida de información.

Teniendo en cuenta lo analizado desde la práctica, extraemos la idea de que los instrumentos de evaluación deben ser variados y explícitos, diseñando para ello un sistema holístico de evaluación que permita realmente valorar en qué medida se ha producido un proceso de aprendizaje colaborativo (Iborra e Izquierdo, 2010). Entre los criterios que estos autores plantean para evaluar dicho proceso destacamos dos que nos parecen fundamentales: 
SONIA CASILLAS MARTÍN, MARCOS CABEZAS GONZÁLEZ Y AZUCENA HERNÁNDEZ MARTÍN

- Considerar todas las dimensiones del saber implicadas: cognitivas, estratégicas, procedimentales, sociales y afectivas.

- Valorar tanto el proceso mismo de aprendizaje, teniendo en cuenta el ritmo y calidad de los logros alcanzados por los grupos, como los resultados o productos de dicho aprendizaje:

El proceso es importante porque permite comprender el acto creativo y social del grupo-alumno, para retroalimentar su actividad y valorar su efectividad. Complementariamente, el producto es necesario en la medida que garantiza la planificación del proceso, es decir, asegura que éste tenga una meta definida, unos objetivos establecidos y unas estrategias para su realización y control (Iborra e Izquierdo, 2010, 227-228).

\subsection{Calidad de las tareas e interés de los recursos digitales}

Teniendo en cuenta lo recogido por los docentes en sus guías de seguimiento, la calidad de las tareas realizadas es valorada de forma muy positiva, tanto por los profesores como por los alumnos, al igual que los recursos digitales empleados, considerados de gran interés y utilidad.

Las actividades propuestas son sencillas, pero coherentes; ajustadas a la edad y capacidades de los estudiantes, y adecuadas a los objetivos de aprendizaje de la asignatura. Sirven para trabajar de forma colaborativa los contenidos y beneficiarse de las aportaciones de los compañeros de grupo, además de promover el interés continuado por las actividades escolares. Y, en síntesis, favorecen el hecho de que los alumnos muestren un mayor interés frente a otros planteamientos centrados en el libro de texto.

Los medios digitales utilizados son variados y, en casi todos los casos, gratuitos y de acceso abierto en la Red: blogs, sitios web, Voki, ClassDojo, Popplet, Screencast-O-Matic, Vocaroo, KidblogyWix. En cuanto a herramientas colaborativas destaca la utilización de los servicios de Google (Google Drive, Youtube) y Edmodo. Y los dispositivos más empleados son los ordenadores, las cámaras de fotografía digitales, teléfonos móviles inteligentes y tabletas digitales.

Lo interesante de todas estas actividades radica en que los estudiantes se posicionan de manera activa en la producción de las mismas y en el hecho de que para desarrollarlas es preciso que empleen distintas herramientas basadas en la utilización de las Tic. La propia naturaleza de las tareas y de los recursos digitales empleados permite que estos pregunten, debatan, diseñen planes, experimenten, recopilen información, analicen datos, saquen conclusiones, comuniquen y compartan ideas con los compañeros, entre otras actividades (Rodríguez y Escofet, 2006; Badía y García, 2006). Y todo ello posibilita la generación de un aprendizaje contextualizado y no abstracto. 


\subsection{Valoración del proyecto por parte de los estudiantes}

Esta valoración se realizó, tal como ya hemos señalado, por medio de un diferencial semántico de 20 ítems con una escala de 1 a 7 . Teniendo en cuenta las respuestas de los 253 alumnos que participaron en los diferentes proyectos, concluimos señalando que los estudiantes valoraron muy positivamente los proyectos en los que participaron en sus respectivos centros. En todos los enunciados se alcanzan puntuaciones elevadas, muy próximas o superiores a 6. Especialmente resaltar que las puntuaciones más altas son las que se refieren al rol de los profesores, reconociendo que les han explicado claramente lo que tienen que hacer, han indicado si realizaban bien las tareas durante su desarrollo y les han informado sobre lo que estaba bien o mal del trabajo. Así mismo afirman que los proyectos les han sido útiles y que han comprendido las actividades propuestas por sus profesores.

Si consideramos los datos sobre las cuatro dimensiones que se establecieron en el cuestionario mediante el análisis factorial -satisfacción, organización, creación y comprensión-, podemos apreciar que las puntuaciones obtenidas por los estudiantes en estos factores son nuevamente muy elevadas, lo que refrenda la valoración positiva a la que anteriormente aludimos. Aunque todas las dimensiones son muy bien valoradas, se puede observar que la comprensión es la que obtuvo mayor puntuación, mientras que la creación fue la dimensión menos estimada.

TABLA 2

VALORACIÓN DE LOS PROYECTOS POR LOS ESTUDIANTES DE ACUERDO A LAS DIMENSIONES OBTENIDAS EN EL ANÁLISIS FACTORIAL

\begin{tabular}{|c|c|}
\hline NOMBRE DEL FACTOR & MEDIA GLOBAL \\
\hline Satisfacción & 6.13 \\
\hline Organización & 6.17 \\
\hline Creación & 5.88 \\
\hline Comprensión & 6.37 \\
\hline Valoración global & 6.13 \\
\hline
\end{tabular}

La propia naturaleza del trabajo colaborativo y el aliciente que conlleva la mediación tecnológica provoca una mayor interacción entre los estudiantes y la dedicación de un tiempo adicional a pensar acerca de las diversas cuestiones planteadas durante el proceso, con lo que el nivel de profundización en dichas cuestiones necesariamente aumenta. 
SONIA CASILLAS MARTÍN, MARCOS CABEZAS GONZÁLEZ Y AZUCENA HERNÁNDEZ MARTÍN

\subsection{Valoración del proyecto por parte de los profesores}

Los proyectos están muy bien considerados. Cuando los profesores se autoevalúan manifiestan sentirse bastante satisfechos con lo realizado. Además, perciben que el alumnado desarrolla actitudes muy positivas, tanto hacia la metodología de aprendizaje colaborativo, como hacia sus compañeros y a ellos como docentes.

Los aspectos más apreciados, que coinciden con los señalados por otros autores como Lou, Abrami, Spence, Poulsen, Chambers y D'Apollonia (1996), y Arancibia, Carcamo, Contreras, Scheihing y Troncoso (2014) son:

- El hecho de que las actividades sirven para trabajar los contenidos.

- Se preparan los materiales y recursos con tiempo suficiente.

- Los materiales didácticos, los elaborados por los profesores y los tecnológicos resultan útiles.

- Los estudiantes están más motivados por aprender porque se ha fomentado el trabajo en grupo y construido conjuntamente el conocimiento, al indagar, investigar y profundizar más en los distintos temas trabajados.

- La evaluación se corresponde con lo que tenían pensado hacer y los alumnos con más capacidades aprovechan las actividades.

Por el contrario, los aspectos menos valorados, también señalados en otras investigaciones como la de Cabero y Marín (2014) y García-Valcárcel y Basilotta, López (2014), se refieren a que la planificación, ejecución y valoración de la tarea les lleva mucho esfuerzo y tiempo; y a los alumnos con más dificultades les sigue costando conseguir los objetivos propuestos.

\subsection{Competencias adquiridas por los estudiantes}

Los profesores manifiestan que los alumnos adquieren competencias conceptuales, procedimentales y actitudinales, si bien predominan las dos últimas. En definitiva, se promueven actitudes muy favorables hacia el aprendizaje, los profesores y el resto de compañeros.

A partir del análisis de las hojas de seguimiento cumplimentadas por los profesores durante el desarrollo del proyecto, podemos extraer algunas competencias adquiridas por los alumnos, de acuerdo con lo que los profesores consideran que ha supuesto trabajar en el contexto de una metodología de aprendizaje colaborativo.

Tanto los alumnos con mayores capacidades como aquellos que presentan dificultades se ven favorecidos con la adopción de acciones pedagógicas de carácter colaborativo mediadas por TIC. Los primeros porque aprovechan mucho las actividades realizadas, y los segundos porque, al trabajar de forma colaborativa, consiguen los objetivos establecidos. Resumiendo, esta metodología de trabajo es provechosa para el proceso de enseñanza y aprendizaje, aunque algunos 
profesores tienen sus dudas sobre si la misma se puede poner en práctica con grupos en los que existan problemas de rendimiento o de conducta.

Aunque se desarrollan competencias conceptuales y procedimentales, los docentes señalan la consecución en mayor medida de las segundas sobre las primeras. Así, por ejemplo, estos aluden especialmente el desarrollo de las que tienen que ver con el vocabulario, la mejora de la expresión, la comunicación y el conocimiento de reglas gramaticales. Se desarrollan, además, estrategias de búsqueda y organización de la información digital, y una mayor capacidad para seleccionar material de forma coherente. Por lo que a las competencias conceptuales se refiere, los estudiantes obtienen conocimientos y conceptos más globales de los temas tratados.

Si consideramos la valoración general del trabajo de todos los grupos, se aprecia cómo el desarrollo de competencias actitudinales se acentúa en todos los casos estudiados. En concreto, podemos destacar, a partir del estudio empírico realizado, las siguientes:

- La cooperación entre los alumnos.

- La responsabilidad individual a la hora de realizar cada uno su parte y aceptar críticas.

- La capacidad para resolver conflictos en momentos de desacuerdo respecto a la tarea realizada.

En consecuencia, el trabajo en grupo, la cooperación, la mayor capacidad para la resolución de problemas y una mejor organización del tiempo y espacio son competencias que se ven favorecidas con el desarrollo de un proceso colaborativo mediado tecnológicamente.

\subsection{Competencias profesionales de los docentes}

La competencia más desarrollada es de tipo procedimental ya que es necesario conocer muy bien el proceso de puesta en práctica de la metodología colaborativa, además de estar al día sobre el empleo técnico y didáctico de las herramientas digitales. Junto a esta competencia, los docentes desarrollan otras de tipo actitudinal, como la motivación por su actividad profesional, la actualización continua, la reflexión, la evaluación y el autoaprendizaje; la disposición para trabajar colaborativamente con otros compañeros, y el interés en la búsqueda de nuevas formas de trabajo que beneficien un tipo de aprendizaje más activo y colaborativo por parte de los alumnos.

Tal como señalan otros trabajos sobre el tema, como el de Gisbert y Lázaro (2015), es esencial la idea señalada por algunos docentes que participaron en los distintos casos sobre la idoneidad de que, desde los propios centros educativos, y considerando sus necesidades e intereses, se promueva un sistema de formación 
SONIA CASILLAS MARTÍN, MARCOS CABEZAS GONZÁLEZ Y AZUCENA HERNÁNDEZ MARTÍN

permanente a través de cursos, seminarios, proyectos de innovación y mejora, o grupos de trabajo.

\section{CONCLUSIONES}

Para finalizar vamos a dar respuesta a una serie de cuestiones directamente relacionadas con las preguntas temáticas que nos planteamos para analizar los distintos proyectos colaborativos. Estas respuestas, dada la naturaleza de nuestro estudio, no pretenden ser generalizables a otros contextos, pero sí pueden ser indicios teóricos extraídos del estudio de prácticas de trabajo colaborativo mediado por el empleo de la tecnología. Retomamos, por tanto, lo que constituía la segunda parte del título de nuestro trabajo: Generando teoría desde el análisis de prácticas educativas.

\section{1. ¿Se dan las condiciones adecuadas en los centros educativos para desarrollar proyectos de trabajo colaborativo por medio de TIC?}

Del análisis de estos casos se desprende cuáles deberían de ser los escenarios y ambientes más propicios para poner en marcha buenas prácticas educativas de carácter colaborativo, mediante el empleo de las herramientas tecnológicas. Hemos puesto de manifiesto cómo en algunos centros existe implicación y apoyo del equipo directivo y de las familias; un respaldo de la administración local y regional, e infraestructuras tecnológicas suficientes y adecuadas. En otros, sin embargo, no se dan las condiciones suficientes porque falta implicación del equipo directivo, de los profesores y de las familias; se aprecia una escasa formación del profesorado $\mathrm{y}$, en ocasiones, las infraestructuras y los medios tecnológicos son insuficientes y/o están desfasados.

Por tanto, es precisa una cultura del centro en donde la construcción de conocimiento colaborativo mediado tecnológicamente implique la participación activa de todos sus protagonistas, el fortalecimiento de la participación y la existencia de los medios adecuados a cada condición específica. En concreto, para que podamos hablar de un proceso de enseñanza y aprendizaje significativos, mediante la puesta en marcha de este tipo de proyectos, son necesarias, al menos, las condiciones que señalamos seguidamente:

- El apoyo real de la administración educativa para dotar de espacios, tiempos y recursos a los centros, de modo que todos los agentes implicados en este tipo de proyectos sientan que pueden acometer con éxito formas de trabajo colaborativo utilizando las herramientas tecnológicas.

- El impulso del equipo directivo, quien debe considerar el proyecto como una apuesta de todo el centro y, en consecuencia, colectivo e integrado.

- La conformación paulatina de un equipo docente con una clara filosofía colaborativa que, además de la apuesta por diseñar e implementar este 
tipo de proyectos en el aula, trabaje a partir de sus propias necesidades e intereses.

- La colaboración estrecha y buscada entre los docentes y las familias. No solo se trata de proporcionarles información sobre lo que se está haciendo, sino también, y dependiendo del nivel educativo y del proyecto en cuestión, solicitar su participación activa.

- La formación docente permanente, tanto en lo que respecta a los modos de trabajo colaborativo, como en lo concerniente a las posibilidades de empleo y tratamiento de las TIC. En este sentido, la fórmula que consideramos más adecuada, en la línea de lo que venimos expresando, es la formación en el propio centro y el establecimiento de comunidades presenciales o virtuales intercentros, en las que participen profesores con intereses comunes.

- La adopción constante de una perspectiva reflexiva y crítica sobre el propio proyecto y otros que se vayan desarrollando, mediante la valoración del mismo por parte de todos los agentes implicados; tanto en términos de procesos como de resultados de aprendizaje y rentabilidad educativa general.

\section{2. ¿Cómo construyen los alumnos el conocimiento a través de la interacción con sus compañeros?}

Los estudiantes aprenden de manera constructivista y por descubrimiento. El conocimiento se genera gracias a espacios de aprendizaje constructivo, a partir de la interacción entre los alumnos y el profesor. Los primeros han de ser los encargados de crear los contenidos utilizando la tecnología, y para ello es preciso que los segundos adopten un papel de mediadores o guías de los aprendizajes, estructurando las actividades de modo sistemático, para que los alumnos puedan trabajar de manera autónoma. La interacción y una asunción clara de roles por parte de los intérpretes implicados en estos proyectos aseguran, junto a otras variables, una construcción conjunta del conocimiento que puede generar aprendizajes ricos y duraderos. Y en este modo de construir el conocimiento y, en definitiva, de aprender haciendo con otros, destaca también la mayor motivación por parte de los estudiantes, quienes otorgan un significado a los contenidos y tareas sobre las que trabajan, porque son cuestiones y problemas significativos para ellos.

\section{3. ¿Adquieren todos los alumnos las competencias deseadas como resultado de los proyectos desarrollados?}

Las estrategias de aprendizaje colaborativo sí favorecen a los alumnos, ya que todos se benefician al construir conjuntamente el conocimiento; compartir responsabilidades; profundizar más en las ideas; tener mayor autonomía y control sobre su propio aprendizaje, y ayudarse unos a otros. El trabajo colaborativo en 
un entorno tecnológico desarrolla en todos los estudiantes un mejor autoconcepto, autonomía, habilidades sociales de diversa índole, mejorando, en general, su rendimiento académico (Carrió, 2007; Arancibia, Carcamo, Contreras, Scheihing y Troncoso, 2014). Concretando aún más las competencias que se pueden potenciar como resultado de la implementación de dichos proyectos, es importante aludir a la generación de operaciones mentales que, sin desdeñar la importancia de la memorización, suponen un mayor nivel de abstracción y al desarrollo de estrategias de autoaprendizaje tales como la reflexión individual, la búsqueda activa y selectiva de información desde múltiples fuentes, la autoevaluación, etc., resultado de algunos roles que los alumnos ejercen en el contexto del proyecto, y entre los que destacamos el de la responsabilidad social asumida.

\section{4. ¿Interesa adaptar la evaluación del trabajo colaborativo para valorar el nivel de aprendizaje de cada estudiante?}

Para comprobar realmente el rendimiento académico a nivel individual y grupal, de tal manera que se pueda valorar con claridad qué competencias concretas se han adquirido, es preciso que el tipo de evaluación esté muy especificado y elaborar instrumentos que permitan a los docentes comprobar en qué medida se han alcanzado las metas del grupo y qué han aprendido o qué tipo de competencias ha adquirido cada alumno, a partir de su participación para conseguir los fines conjuntos planteados. Por todo ello, sí interesa realizar una adaptación de la evaluación del trabajo colaborativo, de modo que al mismo tiempo que se valoren los productos, en términos de competencias alcanzadas por cada miembro y por todo el grupo implicado, se lleve a cabo una valoración de todo el proceso, analizando la propia dinámica grupal. Y ello exige efectuar un ejercicio de autorreflexión colectiva, de autocrítica durante y después del desarrollo del proyecto colaborativo, en el que estén implicados todos los artífices del mismo.

Concluimos considerando la necesidad de planificar de forma muy cuidadosa el trabajo colaborativo, siendo conscientes de sus fortalezas y limitaciones, pero contemplando con optimismo sus posibilidades. Estas constituyen un incentivo suficiente para que los docentes centren sus esfuerzos en desarrollar planteamientos de trabajo con los alumnos en los que se aprovechen las posibilidades que ofrecen las TIC, como entornos comunicativos donde poder construir un conocimiento conjunto y compartido. Tal como evidencian distintas investigaciones sobre el tema (Badía, Becerril y Romero, 2010; Fernández y Valverde, 2014; García-Valcárcel, Basilotta y López, 2014), es preciso que paulatinamente desde los centros escolares, y siempre con el apoyo de la administración educativa, se promueva la creación y/o transformación de las prácticas educativas habituales; mediante el desarrollo de proyectos de trabajo colaborativo que fomenten, a través del empleo de la tecnología, espacios de aprendizaje más flexibles, abiertos, motivadores y ricos en estímulos y en fuentes de información para los estudiantes; de forma que 
estos puedan desarrollar todas las competencias que no sólo establece el currículum, sino que exige la sociedad actual.

\section{REFERENCIAS BIBLIOGRÁFICAS}

Arancibia, M.; Carcamo, L.; Contreras, P.; Scheihing, E. y Troncoso, D. (2014) Re-pensando el uso de las TIC en educación: reflexiones didácticas del uso de la web 2.0 en el aula escolar. Albor, 190 (766). Consultado el 10 de mayo de 2015. http://goo.gl/mzM2hf.

BADÍA, A. y GARCíA, C. (2006) Incorporación de las TIC en la enseñanza y el aprendizaje basados en la elaboración colaborativa de proyectos. Revista de Universidad y Sociedad del Conocimiento, 3 (2). Consultado el 25 de junio de 2015. http://goo.gl/ZFyDH6.

BAdíA, A.; BeCERril, L. y ROMERO, M. (2010) La construcción colaborativa de conocimiento en las redes de comunicación asíncrona y escrita (RCAE): una revisión de los instrumentos analíticos. Cultura y Educación, 22 (4), 1-20.

Cabero, J. y Marín, V. (2014) Posibilidades educativas de las redes sociales y el trabajo en grupo. Percepciones de los alumnos universitarios. Comunicar, 42, 165-172.

Cabezas, M. y Casillas, S. (2009). La Web 2.0: Contexto pedagógico y utilidades didácticas. Papeles Salmantinos de Educación, 13, 247-266.

CARRIó, M. L. (2007) Ventajas del uso de la tecnología en el aprendizaje colaborativo. Revista Iberoamericana de Educación, 41, 1-10.

CeBrián, M. (2008) La web 2.0 como red social de comunicación e información. Estudios sobre el Mensaje Periodístico, 14, 345-361.

Coll, C.; Maurí, T. y Onrubia, J. (2008) Análisis de los usos reales de las TiC en contextos educativos formales: una aproximación socio-cultural. Revista Electrónica de Investigación Educativa, 10, 1-18.

De la Torre, A. (2006) Wededucative 2.0. Edutec, Revista Electrónica de Tecnología Educativa, 20. Consultado el 11 de diciembre de 2014. http://goo.gl/XV0iOe.

Domingo, M.; SÁNCHEZ, J. A. y SANCHO, J. M. (2014) Investigar con y sobre los jóvenes colaborando y educando. Revista Comunicar, 42, 157-164.

DoppenBerg, J. J.; BAKXY, A. y BROK, P. J. (2012) Collaborative teacher learning across foci of collaboration: Perceived activities and outcomes. Teaching and Teacher Education, 28, 899-910.

FernándeZ, M. y Valverde, J. (2014) Comunidades de práctica: un modelo de intervención desde el aprendizaje colaborativo en entornos virtuales. Revista Comunicar, 42, 97-105.

Ferro, C.; Martínez, A. y Otero, M. C. (2009) Ventajas de uso de las tic en el proceso de enseñanza-aprendizaje desde la óptica de los docentes universitarios españoles. Revista Electrónica de Tecnología Educativa, 29. Consultado el 12 de enero de 2012. http:// goo.gl/mHGRZ2.

García-VAlCÁrCel, A. y BasilotTA, V. (2013) How elementary school students evaluate the learning strategy of collaborative projects using ICT, en García, F. J. (ed.) Proceedings of the First International Conference on Technological Ecosystem for Enhancing Multiculturality. New York, ACM, 461-466.

García-VAlCÁrCel, A.; BASIlotTA, V. y LóPez, C. (2014) Las TiC en el aprendizaje colaborativo en el aula de Primaria y Secundaria. Revista Comunicar, 42, 65-74. 
García-Valcárcel, A.; HernándeZ, A. y Recamán, A. (2012) La metodología del aprendizaje colaborativo a través de las TiC: una aproximación a la opinión de profesores y alumnos. Revista Complutense de Educación, 23 (1), 161-188.

García-VAlCÁrcel, A. y Tejedor, J. (2010) Evaluación de procesos de innovación escolar basados en el uso de las TIC desarrollados en la Comunidad de Castilla y León. Revista de Educación, 352, 125-148.

GISBERT, M. y LÁZARO, J. L. (2015) La formación permanente en competencia digital docente y la mejora de la calidad del centro educativo desde la perspectiva de los docentes: un estudio de caso. New Approaches in Educational Research, 4 (2), 124-131.

HERnÁNDEZ, A. y Quintero, A. (2009) La integración de las TIC en el currículum: necesidades formativas e interés del profesorado. Revista Interuniversitaria de Formación del Profesorado, 29 (2-12), 103-119.

IBORRA, A. e IZQuiERDo, M. (2010) ¿Cómo afrontar la evaluación del aprendizaje colaborativo? Una propuesta valorando el proceso, el contenido y el producto de la actividad grupal. Revista General de Información y Documentación, 20, 221-241.

JAVORNIK, M. K. y GRMEK, M. I . (2007) Cooperative learning and team culture in schools: Conditions for teachers' professional development. Teaching and Teacher Education, 24, 59-68.

Johnson, D. W. y Johnson, R. T. (1987) Learning together and alone. Englewood Cliffs, Prentice Hall.

Johnson, D. W.; Johnson, R. T. y Smith, K. A. (1998) Active learning: cooperation in the college classroom. Edina, MN, Interaction Book Company.

LARA, S. (2001) Una estrategia eficaz para fomentar la cooperación. Estudios sobre Educación, 1, 99-110.

lou, Y.; Abrami, Ph.; Spence, J.; Poulsen, C.; Chambers, B. y D'Apollonia, S. (1996) WithinClassgrouping: a meta-analysis. Review of Educational Research, 66 (4), 423-458.

Medina, F. J. y RoBles, A. M. (2012) Enredados: 20 propuestas de aprendizaje cooperativo basadas en la web 2.0. Madrid, Editorial Secretaría General Técnica.

Monereo, C. y BADíA, A. (2012) La competencia informacional desde una perspectiva psicoeducativa: enseñanza basada en la resolución de problemas prototípicos y emergentes. Revista Española de Documentación Científica, 2, 75-99.

Moreno, R. y Martínez, R. (2007) Aprendizaje autónomo. Desarrollo de una definición. Acta Comportamentalia, 15 (1), 51-62.

RodrígueZ, J. L. y Escofet, A. (2006) Aproximación centrada en el estudiante como productor de contenidos digitales en cursos híbridos. Revista de Universidad y Sociedad del Conocimiento, 3 (2). Consultado el 25 de marzo de 2014, http://goo.gl/8CrVtD.

SAlmerón, H.; Rodríguez, S. y GutiérRez, C. (2010) Metodologías que optimizan la comunicación en entornos de aprendizaje virtual. Revista Comunicar, 34, 163-171.

ShaHzad, A.; VAlcke, M. y BAHOO, R. (2012) A Study to Analyze the Teacher's Perceptions About the Adoption of Collaborative Learning in Post-graduate Classes of IUB. Social and Behavioral Sciences, 46, 3056-3059.

Springer, L.; Stanne, M. E. y Donovan, S. S. (1999) Effects of small-group learning on undergraduates in science, mathematics, engineering, and technology: A meta-analysis. Review of Educational Research, 6, 21-52.

STAKE, R. E. (2005) Investigación con estudio de casos. Madrid, Morata.

SuÁrez, C. (2008) Educación y virtualidad. Perú, Editorial Universitaria.

Sú́reZ, C. y Gros, B. (2013) Aprender en red: de la interacción a la colaboración. Barcelona, UOC. 
86 SONIA CASILLAS MARTÍN, MARCOS CABEZAS GONZÁLEZ Y AZUCENA HERNÁNDEZ MARTÍN CONSTRUCCIÓN DE CONOCIMIENTO COLABORATIVO MEDIADO TECNOLÓGICAMENTE: APORTACIONES...

Vázquez-Bernal, B.; Wamba, A.; Jiménez-Pérez, R. y Lorca, A. A. (2010) Percepciones de Futuros Docentes respecto al Aprendizaje Colaborativo Virtual: El caso de Synergeia. Encontro Internacional TIC e Educação, 19 y 20 de noviembre 2010. Consultado el 14 de febrero de 2012. http://goo.gl/z18qaa.

ZABAlA, A. y ARNAU, L. (2009) 11 Ideas clave. Cómo aprender y enseñar competencias. Barcelona, Graó.

ZAÑARTU CORREA, L. M. (2013) Aprendizaje colaborativo. Una nueva forma de relación interpersonal y en red. Consultado el 13 de septiembre de 2016. http://es.slideshare.net/ CeciliaBuffa/luz-mara-zaartu-correa-aprendizaje-colaborativo. 